\title{
État des travaux normatifs dans le domaine du mesurage des radionucléides dans l'environnement
}

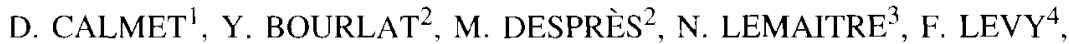 \\ M.C. ROBÉ ${ }^{5}$, J.P. KANCELLARY ${ }^{6}$, P. DIAKONOFF ${ }^{7}$
}

(Manuscrit reçu le 12 juillet 2001, accepté le 20 janvier 2002)

\begin{abstract}
RÉSUMÉ Les préoccupations concernant la présence de substances radioactives dans l'environnement conduisent régulièrement à des expertises sur les niveaux d'activité des radionucléides dans l'environnement et à des évaluations de leurs impacts radiologiques sur les populations humaines. La crédibilité de ces expertises repose en partie sur la qualité et la fiabilité des mesurages. Ce document présente les systèmes d'organisations de la normalisation, nationale et internationale, ainsi que les documents normatifs publiés et les travaux de normalisation en cours relatifs à la métrologie des radionucléides présents dans les matrices environnementales.
\end{abstract}

ABSTRACT Status report on standard-setting work in the area of environmental measurement.

Environmental assessment studies are regularly commissioned to study the activity levels of radioactive substances in the environment and to assess their radiological impact on the public, in response to concern about the presence of such substances. The credibility of such studies relies, in part, on the quality and reliability of measurements. This document describes standardization organization and their systems, both national and international, the standard-setting documents published as well as standardization work under way on the measurement of radionuclides found in environmental matrices.

\section{Introduction}

La préexistence et les ajouts, autorisés ou accidentels, de substances radioactives dans l'environnement justifient les demandes d'expertises sur l'état radiologique de l'environnement (radioécologie) et les évaluations d'impacts sur les populations humaines (radioprotection). Ces questions émanent tant des instances officielles internationales, nationales ou régionales que des exploitants d'installations

\footnotetext{
${ }^{1}$ CEA. Direction Matières Surveillance Environnement, hâtiment DAM, BP 12,91680 Bruyères-le-Châtel, France.

${ }^{2}$ CEA, 91191 Gil-sur-Yvette Cclex, Irance.

${ }^{3}$ OPRI, 31 rue de l'ćcluse, 78110 Lc Vésinet, France.

${ }^{4}$ COCjeMA. BP 170, 30205 Bagnols-sur-Cèzc Cedex, France.

${ }^{5}$ IPSN, 60-68 avenuc du général Leclere, BP 6, 92265 Fontenay-aux-Roses Cedex, France.

'BNEN - EDF/DER, I avenue du général de Gaulle, 92141 Clamart Cedex. France.

${ }^{7}$ AFNOR - UAN/DGIE, 11 avenue Francis de Préssensé, 9357I Saint-Denis-la-Plaine Cedex, France.
} 
nucléaires mais également des médias et des associations publiques locales. De plus, la prise de conscience de l'enjeu que représente la protection de l'environnement aboutit à une demande de référentiels qui permettent aux acteurs socio-économiques d'évaluer leurs activités vis-à-vis de l'environnement (International Standard Organisation, 1996). Dans ce contexte, même si actuellement les niveaux de radioactivité attendus dans l'environnement sont faibles, des résultats de mesurages sont requis pour établir l'impact des pratiques ou des accidents nucléaires en cause (National Radiological Protection Board, 1995). Les résultats de ces mesurages sont utilisés pour établir des états radiologiques ponctuels, des chronologies sur le long terme ou pour expliciter et quantifier les mécanismes de transfert des radionucléides entre les différents constituants de l'environnement. Ils seront éventuellement confrontés aux résultats des calculs, obtenus par des simulations mathématiques, afin de valider les évaluations théoriques d'impacts et de risques. La crédibilité des expertises dans le domaine «nucléaire et environnement » repose donc, en partie, sur la qualité et la fiabilité des mesurages de radionucléides, comme l'indiquait plus généralement un rapport de l'Académie des sciences qui spécifiait que «les questions de mesure occupent une place centrale dans le domaine de l'environnement » (Académie des sciences, 1996).

Depuis la découverte des rayons $\mathrm{X}$ et de la radioactivité à la fin du siècle dernier, la métrologie des rayonnements ionisants s'est développée parallèlement aux applications médicales, industrielles et militaires. Le mesurage d'un radionucléide, présent dans un échantillon de l'environnement, consiste à déterminer la grandeur « activité » dont l'unité est le becquerel. Un ensemble de grandeurs et d'unités spécifiques de la métrologie des rayonnements ionisants a ainsi été défini et les moyens nécessaires à la réalisation des étalons et des raccordements métrologiques nécessaires aux différentes applications ont été mis en place dans la plupart des pays industrialisés. Aujourd'hui, la tendance est à l'internationalisation du contrôle et de la surveillance des activités et des pratiques industrielles à travers l'adoption de traités ou de conventions internationales de protection de l'environnement. Elles requièrent l'évaluation de l'impact potentiel des contaminations transfrontières, chroniques ou accidentelles.

Pour l'industrie nucléaire et son rapport à l'environnement, cet élargissement des partenaires nationaux à ceux d'autres états impose que les résultats des mesurages d'activités, présentés par un pays pour caractériser des effluents rejetés dans l'environnement ou des échantillons environnementaux, soient fiables et reproductibles pour être acceptés par l'ensemble des états potentiellement concernés par une contamination régionale transfrontière.

Aujourd'hui, le niveau de développement des techniques de mesure nucléaires utilisées pour le mesurage des radionucléides a dépassé le stade de la maturité 
(Laitinen, 1973) car les mécanismes en jeu sont bien compris, les principes établis et des équipements performants sont disponibles (National Council on Radiation and Measurements, 1994). Ce degré de développement se traduit, entre autres, par l'existence de documents normatifs décrivant les protocoles de mesurage (International Standard Organisation, 1989, 1992, 1997). Les méthodologies, y compris les parties concernant les protocoles radiochimiques, et les différentes techniques de mesure nucléaires ont été adaptées aux matrices environnementales: gaz, aérosols, eaux naturelles, sols, sédiments, organes de végétaux ou d'animaux considérés comme bioindicateurs. C'est ce même découpage qui a été retenu pour le programme 135 ; « Analyse des radionucléides dans l'environnement" du Comité français d'accréditation (COFRAC) pour l'accréditation des laboratoires d'essais dans ce domaine. Cette approche est en accord avec celle des études et expertises nécessitant une évaluation d'impact radiologique sur l'environnement ou sur les populations.

Les méthodologies et les installations de mesurages doivent également être adaptées aux développements et modifications des pratiques liées à l'évolution des applications nucléaires qui conduisent à la baisse progressive des rejets de radionucléides dans l'environnement. Aujourd'hui, les niveaux d'activités des radionucléides artificiels au sein des différentes composantes aquatiques et terrestres de la plus grande partie du territoire français, relèvent du domaine de mesure des traces 8 , voire des ultra-traces. Dans tous les cas, les méthodologies retenues doivent être conformes aux exigences des normes ISO pour les laboratoires d'essai (International Standard Organisation, 1990) afin de garantir la fiabilité des résultats de mesurage nécessaires à la crédibilité des études scientifiques, expertises ou des contrôles réglementaires.

Ce document présente brièvement les travaux de normalisation et les règles de raccordement nécessaires en métrologie des radionucléides présents dans les matrices environnementales en croisant les trois niveaux : organisations nationales françaises, européennes et internationales de normalisation. Il fait également le bilan des documents normatifs en vigueur dans ce domaine.

\section{Mesurage des radionucléides dans l'environnement}

Les expertises qui portent sur des radionucléides d'origine naturelle concernent généralement des remobilisations d'éléments qui conduisent à des ajouts de faibles quantités d'activité par rapport à leurs niveaux naturels. En ce qui concerne les

\footnotetext{
${ }^{8}$ De nombreux analystes utilisent le terme de traces pour décrire des dérerminarions réalisées à des niveaux inférieurs à une partie par million (ppm), d'autres l'utilisent pour des analyses où l'analyte est à des niveatux de concentrations très laibles qui posent des diflicultés techniques pour l'obtention de résultats valicles.
} 
expertises sur l'activité des radionucléides artificiels, celle-ci est généralement très inférieure à celles des autres constituants naturels radioactifs de la matrice. De plus, des contaminations provenant de réactifs, des équipements ou de l'environnement du laboratoire peuvent conduire à des résultats surestimés. En outre, les pertes de l'analyte à mesurer, lors des opérations de préparations radiochimiques ou autres, sont plus critiques aux faibles concentrations ou lors de la recherche d'écarts d'activité faibles et peuvent conduire à être dans l'incapacité de détecter l'analyte ou sa variation d'activité. Enfin, l'interférence des constituants de la matrice avec le système de détection peut conduire à des concentrations sous-estimées ou surestimées qui nécessitent de rajouter des étapes de purification ou l'utilisation de détecteurs plus sélectifs.

Pour répondre à ces contraintes, le métrologiste doit donc abaisser les limites de détection et augmenter la précision et la justesse des mesurages. Dans ce contexte, garantir la fiabilité des résultats obtenus est considéré comme la question majeure de l'analyse des radionucléides dans les matrices environnementales (Prichard et al., 1998). Ces questions font l'objet de publications régulières (Ibrahim et al., 1995 ; Nir-El, 1998 ; Parkinson et Horril, 1984 ; Sima et Dovlete, 1997) et conduisent parfois à la mise en cause des procédures de mesurage des radionucléides ou des étalons utilisés, souvent inadaptés aux niveaux des activités trouvés actuellement dans l'environnement et non représentatifs de la composition élémentaire des matrices environnementales. La seule réponse possible est la mise en place d'un système d'assurance de la qualité rigoureux qui respecte les bonnes pratiques de laboratoire (OECD, 1992) et les exigences énoncées dans les principes de l'assurance de la qualité, codifiées dans les documents de la série dé l'ISO 9000 et 9001 pour la certification et du guide ISO/CEI $n^{\circ} 25$ et de la norme ISO 17025, pour l'accréditation.

\section{Organisation de la normalisation dans le domaine nucléaire}

Le Bureau de normalisation des équipements nucléaires (BNEN) et l'Association française de normalisation (AFNOR) sont les organisations qui s'occupent au niveau national français de la normalisation en métrologie. Au niveau international, le Comité européen de normalisation (CEN) et l'International Standardization Organisation (ISO) s'efforcent de mettre en place une pratique métrologique rigoureuse pour le mesurage, entre autre, de l'activité des radionucléides afin d'assurer la comparabilité des résultats obtenus dans les différents états membres de ces organisations.

\subsection{Organisation en France}

Au niveau français, agréé le 26 juin 1990 par décision du Ministère de l'industrie et de l'aménagement du territoire, le BNEN est en charge de la gestion des 


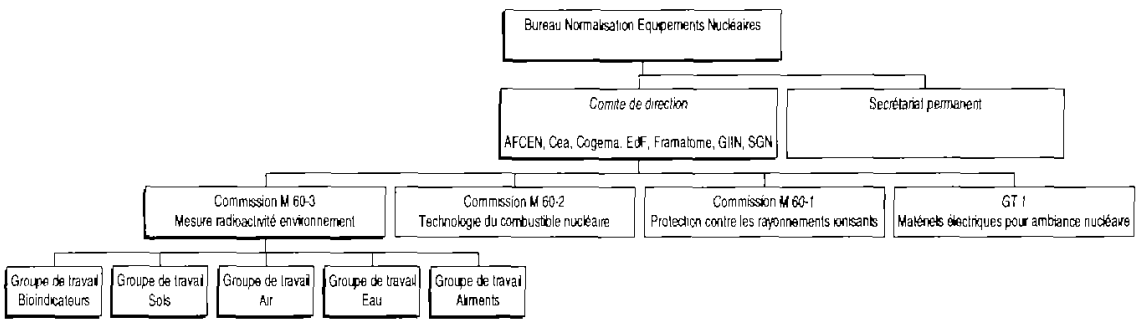

Figure 1 -Organisation du bureau de normalisation des équipements nucléaires dans lequel quatre groupes de travail traitent spécifiquement des méthodologies de mesurage des radionucléides au sein des quatre grands types de matrices environnementales : air, eau, sols et organismes bioindicateurs.

Organization of the Standardization Office for Nuclear equipment with four working groups specifically addressing radionuclide measurement in four major environmental matrices: air, water, soil and bioindicator organisms.

commissions françaises et des groupes de travail associés, dans les domaines de la protection contre les rayonnements ionisants (commission M60-1), de la technologie du cycle du combustible nucléaire (commission M60-2) et du mesurage de la radioactivité dans l'environnement (commission M60-3). Cette dernière commission a été créée en 1992 à la suite de litiges sur des résultats de mesurages obtenus par différents laboratoires, à la demande du Ministère de l'industrie. Celui-ci a donc chargé l'AFNOR de lui proposer des travaux normatifs sur les mesurages de la radioactivité dans l'environnement. L'approche normative semblait pouvoir répondre à une facette des contentieux entre différents organismes publics et privés susceptibles d'émettre des résultats d'activité significativement différents sur des échantillons apparemment identiques.

Pour répondre aux objectifs de l'AFNOR, la commission M60-3 a été structurée en quatre groupes de travail en fonction des principales matrices environnementales : air, eau, sols et bioindicateurs qui répondent également aux divisions du programme 135 du COFRAC. Un cinquième groupe de travail «Aliments » lui a été associé pour répondre aux exigences du programme 99-4 du COFRAC.

Cette commission est chargée de la normalisation des méthodes de mesurage de la radioactivité dans l'environnement, à l'exception de la radioprotection des travailleurs, du domaine médical et de la contamination. Depuis sa création, les travaux de cette commission ont conduit à la rédaction de 37 normes AFNOR sur les méthodes de prélèvements des échantillons de l'environnement et de mesurages des radionucléides. 


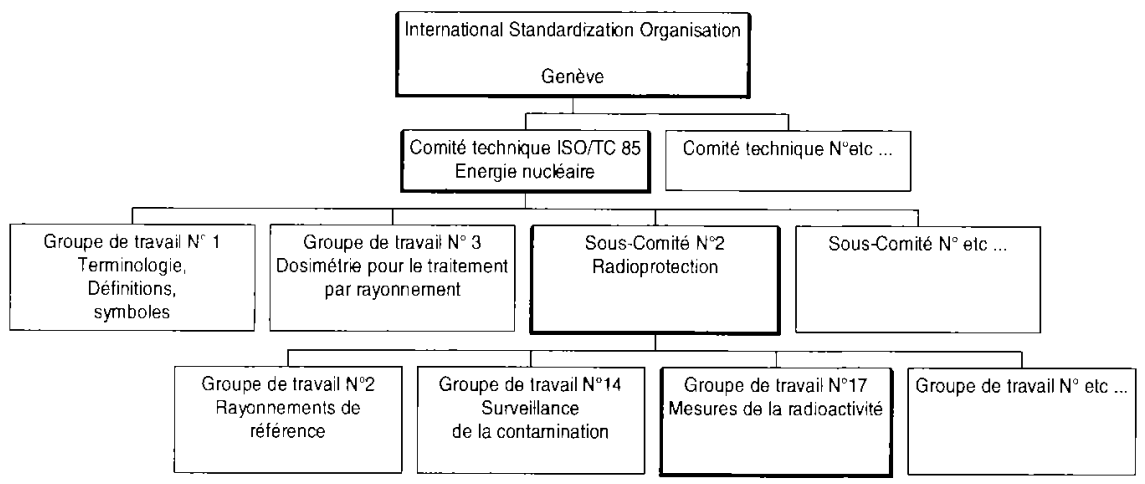

Figure 2 - Organisation du mesurage de la radioactivité au sein des groupes de travail de l'International Standardization Organisation et positionnement du groupe de travail $n^{\circ} 17$ traitant du mesurage de la radioactivité.

Organization of radioactivity measurement in the working groups of the International Standardization Organization and status of working group 17 on measurement of radioactivity.

\subsection{Organisation au niveau international}

Au sein de l'ISO, c'est le comité technique 85 (TC85) chargé des différents aspects de l'utilisation de l'énergie nucléaire qui regroupe, dans le sous-comité $\mathrm{n}^{\circ} 2$ «Radioprotection », les activités de normalisation qui intéressent le mesurage des radionucléides. Depuis septembre 1992, les travaux du TC85, portant sur les limites de détection et le mesurage du radon, ont été rattachés à la commission M60-3. À partir de 1998, c'est au sein du groupe de travail $n^{\circ} 17$ « Mesures de la radioactivité » que sont traitées plus particulièrement les questions de métrologie de la radioactivité de l'environnement. Il est prévu d'y comparer les différentes normes nationales concernant le mesurage de la radioactivité dans l'environnement, émises par les pays européens, France (AFNOR), Allemagne et Pays-Bas ainsi que par les États-Unis d'Amérique (American Society for Testing and Materials, ASTM) afin de les éditer sous la forme de normes ISO. C'est dans ce groupe de travail que les 8 normes AFNOR sur le mesurage de la radioactivité des sols rédigées par la commission M60-3 sont en cours d'examen pour être publiées sous forme de normes ISO.

\section{Documents à caractère normatif}

Les études radioécologiques et les évaluations d'impact sanitaire sont basées sur des résultats de mesurages réalisées sur des échantillons environnementaux et d'aliments prélevés suivant des méthodologies dites génériques qui font l'objet de 
publications internationales (AIEA). Ainsi les vecteurs physiques, air et eau, susceptibles de disperser les radionucléides respectivement, sous forme de gaz ou d'aérosols d'une part et sous forme dissoute ou particulaire d'autre part, ainsi que les composantes naturelles, sol-sédiments, végétaux et animaux où peuvent être stockés les radionucléides suivant un mode transitoire, font l'objet de stratégies et de modes de prélèvements spécifiques. Par contre les approches exploratoires permettant la quantification de la contamination de ces composantes par les radionucléides ne différent pas fondamentalement de celles utilisées pour l'appréciation de contaminations ou de pollutions environnementales par des composés toxiques métalliques ou organiques. Aussi, alors que les techniques de mesures des radionucléides sont parfois spécifiques (mesure des émissions nucléaires), les techniques de prélèvements et de conservation des échantillons environnementaux relèvent d'un «commun » aux études d'impact en général. Dans les paragraphes suivants, nous verrons comment les documents produits par la Commission M60-3 se positionnent dans le contexte des normes AFNOR et ISO déjà publiées, occasionnellement de celles de l'ASTM américaine et de la commission électrotechnique internationale (CEI), plus particulièrement chargée de l'instrumentation et des équipements reposant sur des composants électroniques.

\subsection{Qualité de l'eau}

Les travaux normatifs rédigés par le groupe «Eau », s'adressent aux laboratoires chargés de déterminer la radioactivité présente dans l'eau potable et les eaux brutes peu chargées en sels. L'effort normatif de l'AFNOR, puis de l'ISO, dans le domaine de la qualité de l'eau a été important dans le passé et concrétisé par la publication d'un jeu quasi complet de documents applicables aux expertises et recherches radioécologiques. Ainsi, un ensemble de normes (série 5667 de l'ISO) couvrent les principes généraux de l'échantillonnage et de la conservation des échantillons d'eau au sein des différents systèmes aquatiques dulçaquicoles ${ }^{9}$ et marins. La conservation des échantillons en vue de la mesure des radionucléides a été intégrée dans la norme générale. Des normes particulières ont été élaborées pour le mesurage des indices globaux de la radioactivité alpha et bêta pour les eaux non salines (ISO 9696 et 9697) et pour le mesurage des émetteurs bêta (tritium) par scintillation liquide (ISO 9698) et des émetteurs gamma par spectrométrie gamma haute résolution (ISO 10703).

Des documents complémentaires ont été publiés par la commission M60-3, en particulier pour la détermination des indices de la radioactivité alpha

\footnotetext{
${ }^{9}$ Dulçaquicole : qui concerne uniquement les eaux douces.
} 
D. CALMET et al.

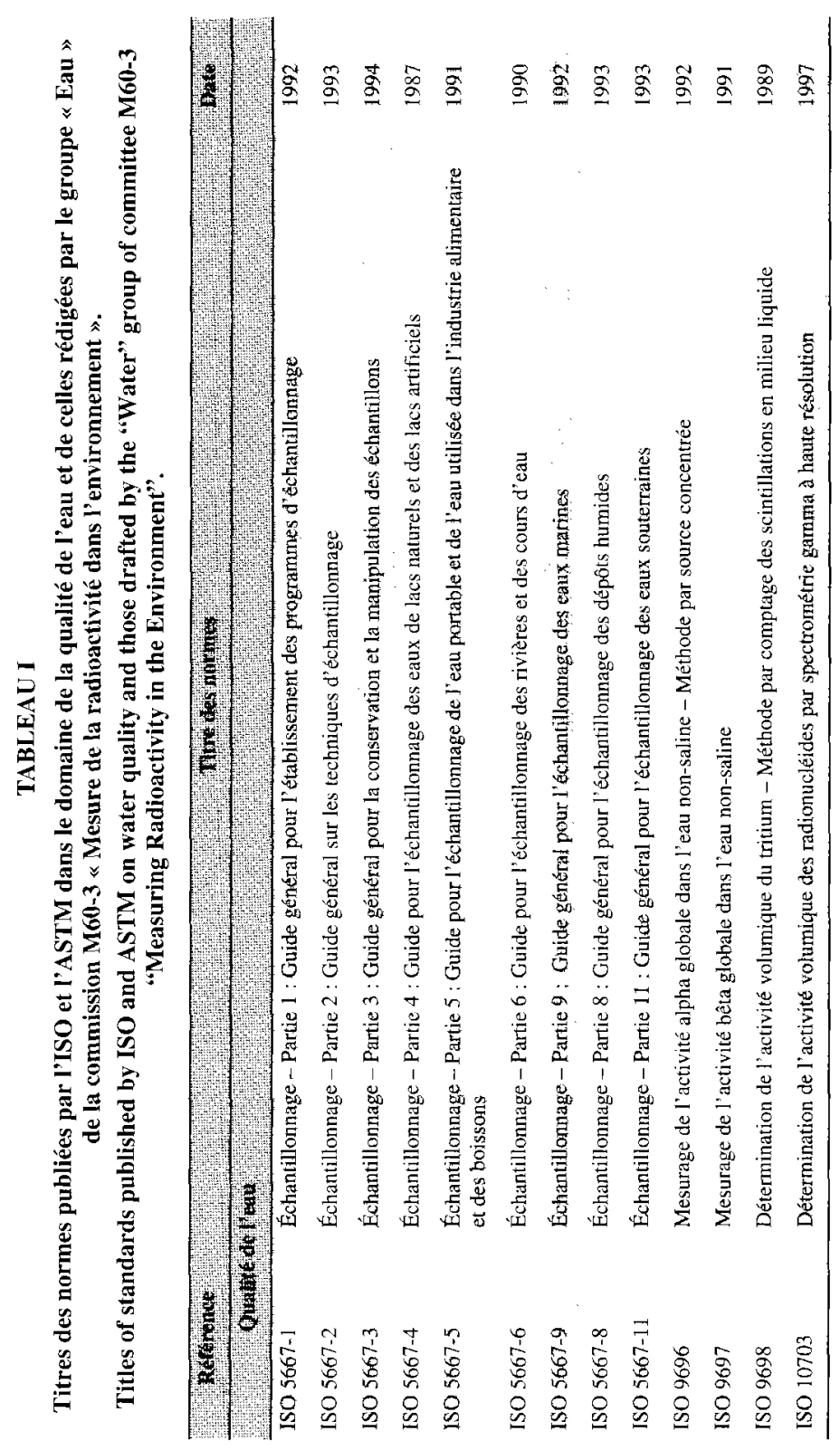


ÉTAT DES TRAVAUX NORMATIFS

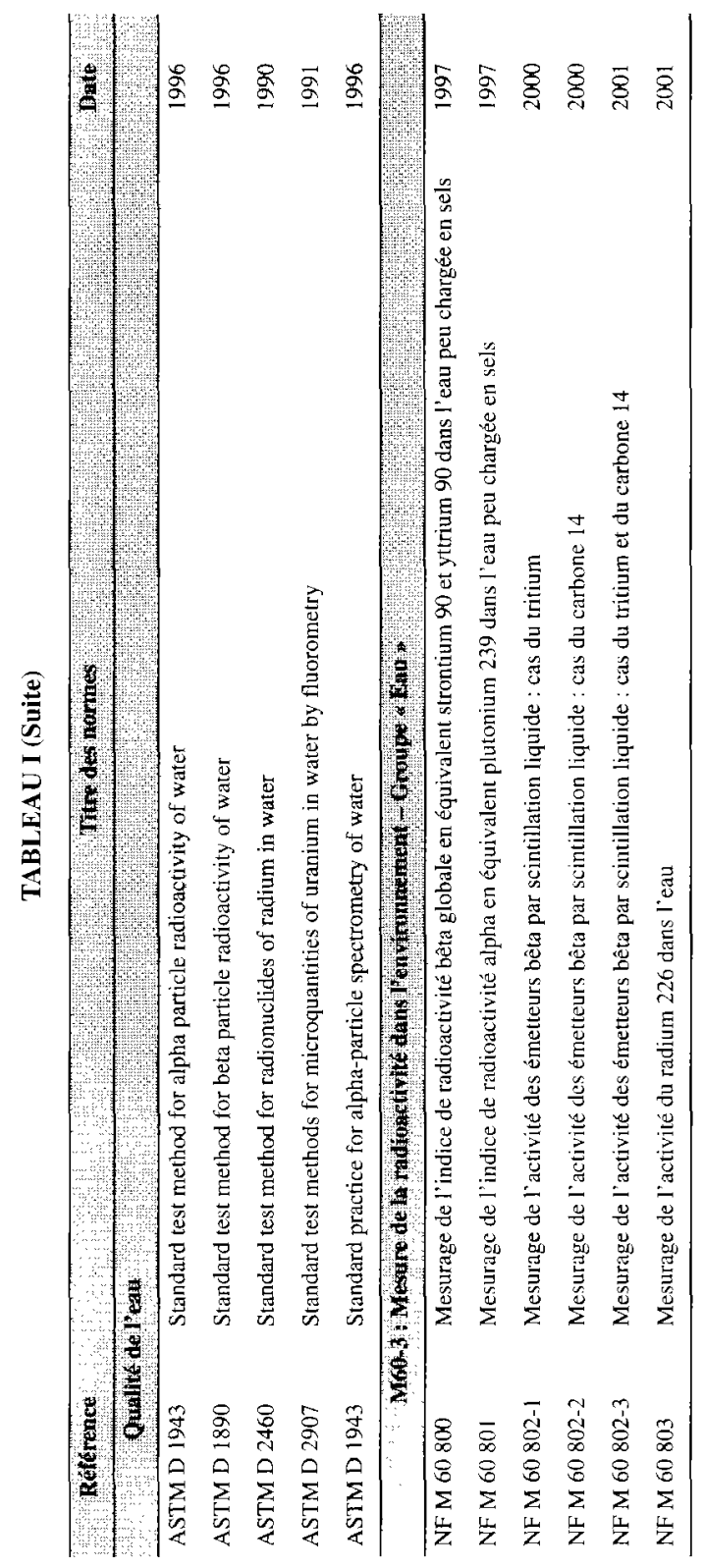

RADIOPROTECTION - VOI. $37-\mathrm{N}^{\circ} \mathrm{I}(2002)$ 
(NF M 60 801) et bêta (NF M 60800 ) des eaux peu chargées en sels, l'actualisation de la norme de mesurage du tritium dans les eaux (NF M 60 802-1) et de celle du mesurage du carbone 14 (NF M60 802-2), puis de ces deux radionucléides simultanément (NF M 60 802-3). En effet, la surveillance des niveaux de tritium fait l'objet d'une attention toute particulière au niveau européen (CCE, 1998) dans les eaux de boisson, justifié par les rejets actuels des installations nucléaires qui sont des sources locales non négligeables de tritium dans l'environnement. La radiotoxicité du tritium est considérée comme faible mais le contrôle de l'activité volumique du tritium dans l'environnement est réalisé pour pouvoir suivre sa circulation dans l'hydrosphère et la biosphère jusqu'aux eaux de boisson.

Une norme a été publiée sur la mesure de l'activité du radium 226 dans les eaux (NF M 60803 ).

Après la révision de la directive $80 / 778 / \mathrm{CCE}$ relative à la qualité de l'eau potable qui a abouti à la directive $98 / 83$ relative à la qualité des eaux destinées à la consommation humaine, l'existence de normes AFNOR sur ce sujet est donc importante pour les discussions techniques qui se tiendront au niveau européen.

\subsection{Qualité de l'air}

L'effort normatif de l'AFNOR et de l'ISO dans le domaine de la qualité de l'air a porté principalement sur les principes généraux de l'échantillonnage des radionucléides présents dans l'air, avec une production dominante de documents concernant ceux associés à la fraction particulaire. La CEI a édité une norme concernant le prélèvement du tritium et son mesurage (CEI 710). Quatre avantprojets concernent l'instrumentation de mesure du radon et de ses descendants (CEI 61577-1, -2, -3, -4).

La commission M60-3 s'est donc engagée dans une révision de la norme sur la détermination volumique du tritium atmosphérique et l'élaboration de normes complémentaires sur le prélèvement des aérosols (M 60 760), la détermination de l'activité des dépôts au sol (M 60770 ) et le mesurage des halogènes. L'importance de l'effort a porté sur le mesurage du radon, gaz radioactif d'origine naturelle résultant de la désintégration de l'uranium et du radium en provenance du sol. Cet élément atteint des niveaux significatifs dans l'air des régions aux sous-sols granitiques et volcaniques et peut également être émis par les matériaux de construction.

Le radon et ses descendants solides sont inhalés et irradient les poumons. Aussi, afin de répondre à la circulaire conjointe DGS et DGUHC n ${ }^{\circ}$ 99/46 du 27 janvier 1999, définissant les actions qui doivent être menées dans la gestion du risque radon dans les locaux, un effort particulier a été réalisé sur les différentes 
ÉTAT DES TRAVAUX NORMATIFS

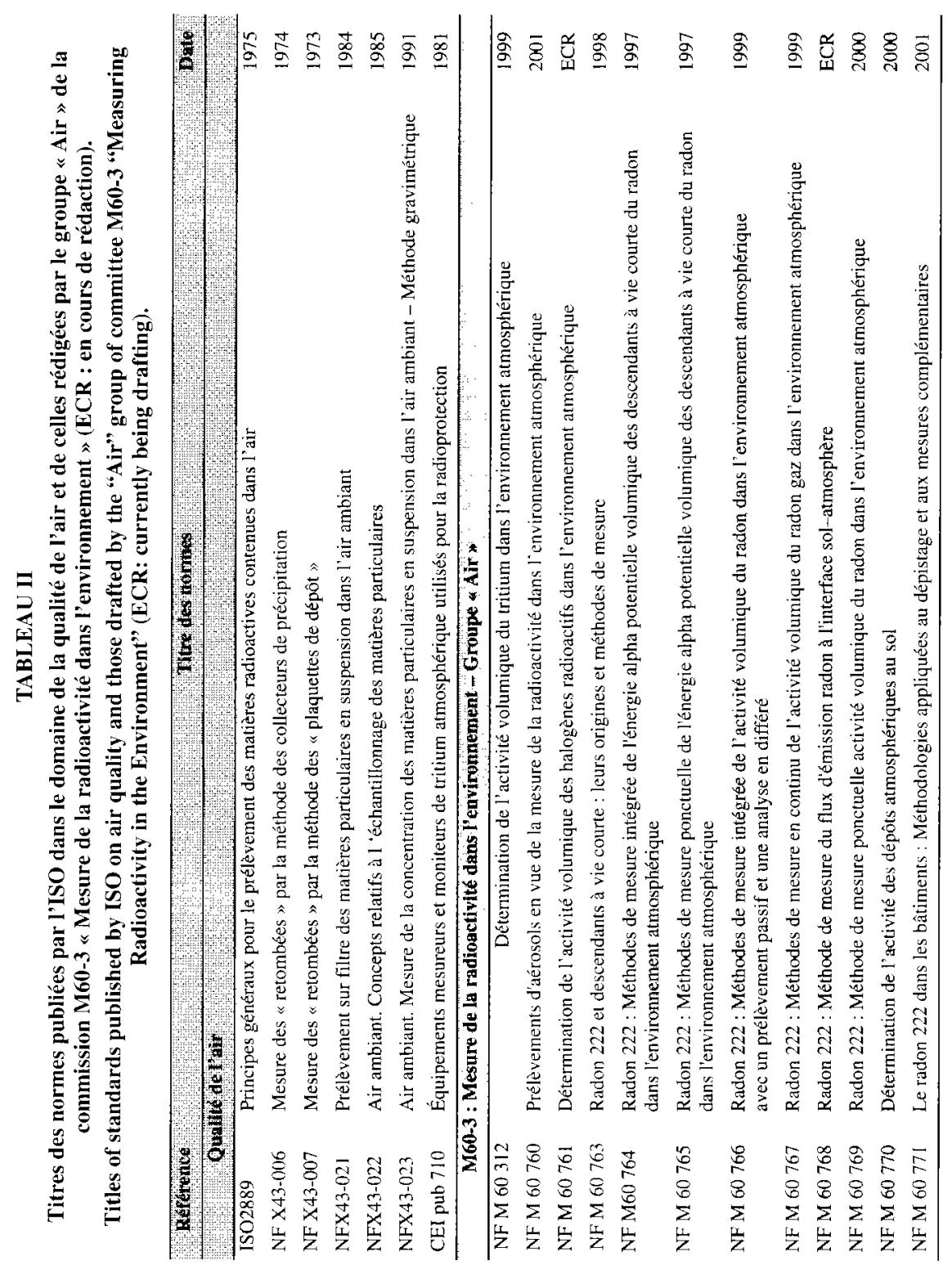


méthodes de mesurage du radon 222 et de ses descendants. Huit normes sur ce sujet ont été publiées (série radon 222).

Toujours sur le thème du radon, il faut noter que la traduction de la norme ISO 13466 en français a été validée par des membres de la commission M60-3. Par ailleurs, dans le cadre des travaux de normalisation de la CEI, des membres de la commission participent au groupe de travail $45 \mathrm{~B}$.

\subsection{Qualité des sols}

Les normes AFNOR et ISO dans le domaine de la qualité des sols concernent les travaux portant sur les principes généraux de l'échantillonnage et de la préparation des échantillons de sols avant détection des radionucléides. Elles s'inscrivent dans la méthodologie d'étude de site, en particulier celle utilisée en radioécologie.

La commission M60-3 a donc repris les documents normatifs existants pour les adapter aux questions posées dans les expertises et études nécessitant des mesurages de radionucléides, en particulier pour les indices de radioactivité alpha et bêta globaux, les émetteurs gamma, le strontium 90 et le plutonium. Cette même approche a été suivie par l'ASTM.

Le groupe de travail « Sol » a donc élaboré 8 normes AFNOR (série M 60790 ). Il s'est efforcé de répondre aux attentes des organismes chargés de déterminer les principaux radionucléides présents dans des sols, dans les cas d'une surveillance de routine, d'une expertise ou d'une caractérisation initiale de site, d'une étude de la distribution verticale des radionucléides dans les sols, ou d'une étude de la radioactivité superficielle des sols, en vue d'étudier les retombées atmosphériques ou la remise en suspension.

La première norme (NF M60-790-1) fixe le cadre général et les étapes à suivre. La deuxième norme est un guide pour la sélection des zones de prélèvements et d'échantillonnage, le transport et la conservation des échantillons de sol (NF M60790-2). Les six autres normes présentent les méthodes de traitement et de mesurage des échantillons de sol en laboratoire. Les normes 790-3 et 790-4 concernent respectivement le séchage, le broyage, le tamisage et l'homogénéisation des échantillons d'une part, et la mise en solution des radionucléides à mesurer, étape nécessaire à la recherche sélective de radionucléides émetteurs bêta purs (comme le strontium 90) et alpha (isotopes 238 , 239 et 240 du plutonium) d'autre part. Le mesurage des indices de radioactivité alpha globale et bêta globale qui permettent de réaliser une première évaluation de la dispersion des niveaux d'activité fait l'objet de la norme NF M60-790-5. Le mesurage des radionucléides émetteurs gamma, en particulier les principaux 
ÉTAT DES TRAVAUX NORMATIFS

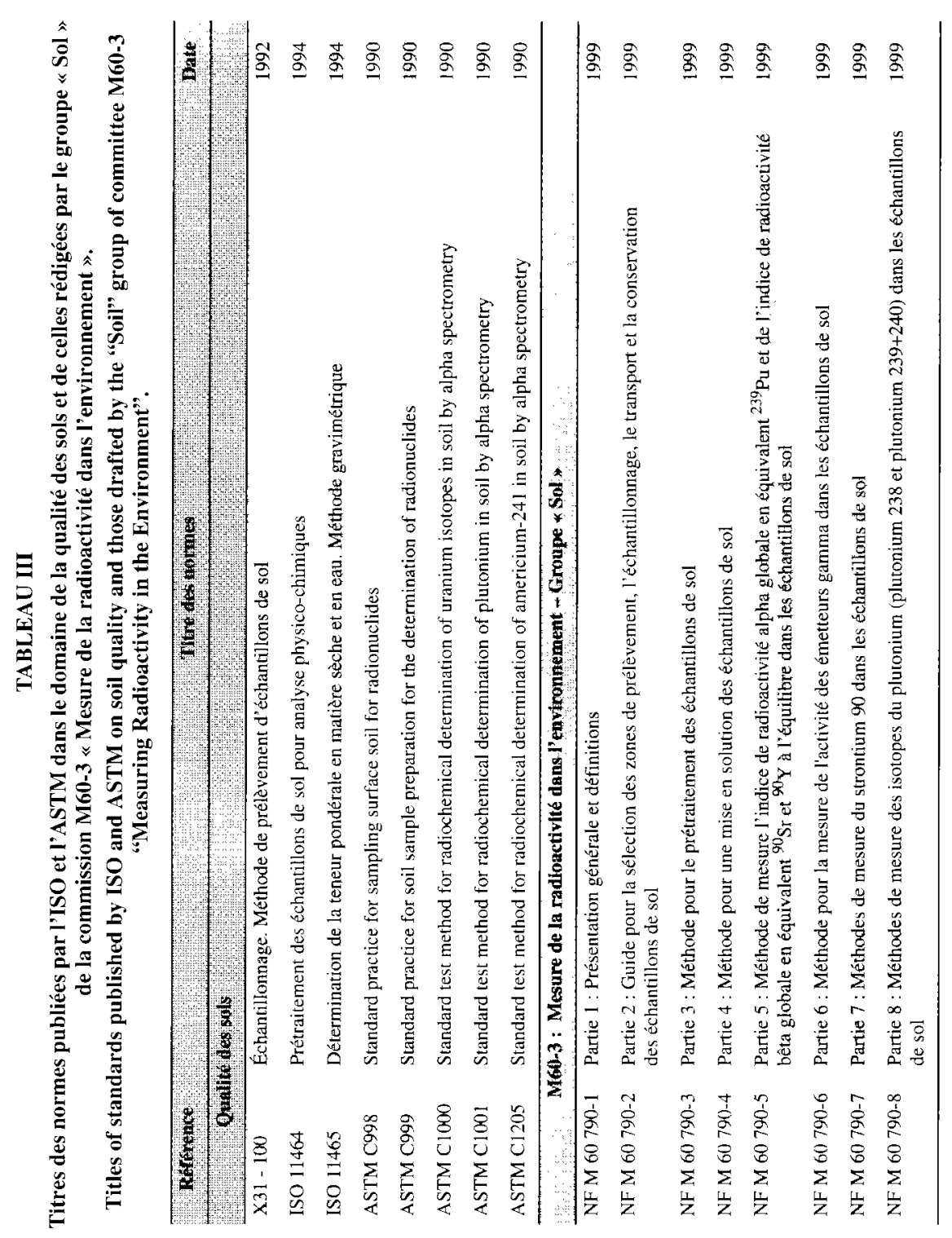


descendants des chaînes naturelles de l'uranium et du thorium et les radionucléides artificiels dont le césium 137 qui est systématiquement présent dans le niveau de surface des sols (retombées des essais nucléaires atmosphériques et de l'accident de Tchernobyl) est traité dans la norme NF M60-790-6. Les parties 8 et 9 de cette série traitent respectivement du mesurage de strontium 90 et des isotopes émetteurs alpha du plutonium (plutonium 238, 239 et 240). Elles font appel à des protocoles opératoires complexes dont l'intérêt est justifié par la radiotoxicité potentielle de ces éléments.

Un travail analogue pour les sédiments marins et aquatiques a été entrepris en 2000 , ainsi que la rédaction d'une norme sur le mesurage direct des radionucléides émetteurs gamma par spectrométrie gamma in situ.

\subsection{Bioindicateurs}

L'utilisation d'organismes vivants, en particulier de bioindicateurs caractérisés par une capacité d'accumulation élevée des contaminants industriels, y compris les radionucléides, permet d'évaluer les évolutions qualitative et quantitative de la contamination des milieux naturels. Les bioindicateurs s'avèrent particulièrement adaptés en tant qu'intégrateurs de toute forme de rejets de radionucléides susceptibles d'avoir un impact sur l'environnement.

En l'absence de précaution pour la conservation des échantillons, les indicateurs biologiques sont susceptibles de se dégrader rapidement entre la date du prélèvement et celle de leur analyse au laboratoire. La conservation des échantillons pendant le transport ainsi que pendant le temps durant lequel les échantillons sont stockés au laboratoire avant d'être analysés, doit garantir que les concentrations déterminées seront représentatives de celles de l'échantillon initial, en particulier pour les éléments majeurs constitutifs de la matière vivante.

L'identification d'un groupe «bioindicateurs » au sein de la commission M60-3, qui n'apparaît pas dans les autres structures normatives, a conduit ce groupe à se rapprocher des normes existantes de l'AFNOR et de l'ISO dans les domaines de la qualité de l'eau, des produits agricoles et des aliments.

Les travaux normatifs ont débouché sur la rédaction de guides généraux sur les précautions à prendre pour le prélèvement, la conservation et la manipulation des échantillons d'indicateurs biologiques avant leur prise en charge au laboratoire (M 60 780-1, -2, -3, -4). Une deuxième série de guides concernant l'échantillonnage d'indicateurs biologiques spécifiques des différents milieux terrestres, dulçaquicole et marin (M 60 780-6, -7, -8) a également été publiée. 
ÉTAT DES TRAVAUX NORMATIFS

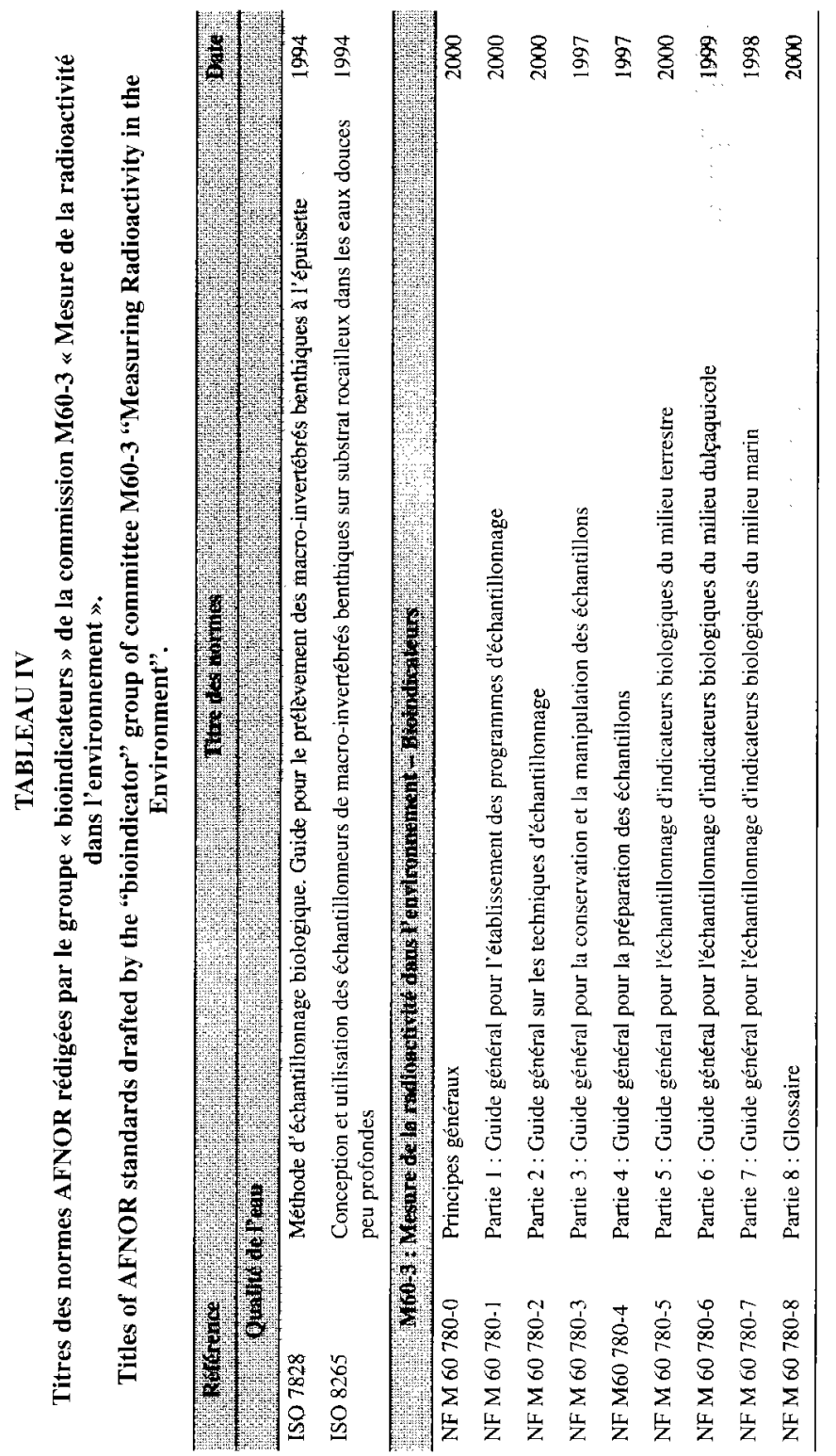

RADIOPROTECTION - VOL. 37 - N $1(2002)$ 


\section{TABLEAU V}

Titres des normes AFNOR rédigées par le groupe aliments de la commission M60-3 « Mesure de la radioactivité dans l'environnement » (ECP : en cours de publication).

Titles of AFNOR standards drafted by the foodstuffs group of committee M60-3 "Measuring Radioactivity in the Environment" (ECP: currently being publishing).

\begin{tabular}{|c|c|c|}
\hline Référence & 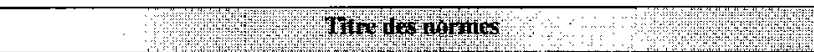 & Date \\
\hline \multicolumn{3}{|c|}{ 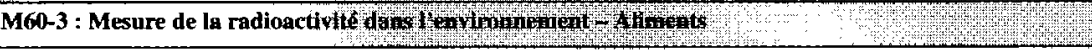 } \\
\hline NF V 03-900-1 & $\begin{array}{l}\text { Partie I : Guide pour l'Échantillonnage, le transport et la conscrvation des denrées } \\
\text { alimentaires - Obtention d'un échantillon pour kaboratoire }\end{array}$ & ECP \\
\hline NF V $03-900.2$ & $\begin{array}{l}\text { Partie } 2 \text { : Guide pour la préparation des denrées alimentaires - Obtention } \\
\text { d'un Échantillon pour essai }\end{array}$ & $\mathrm{ECP}$ \\
\hline
\end{tabular}

\subsection{Aliments}

Pour la partie «Aliments », au plan français, les travaux prévus par le groupe de travail du BNEN ont été présentés au comité d'orientation stratégique (COS) agroalimentaire. Le programme a conduit la rédaction de deux nouveaux projets de normes qui viennent compléter celles produites sous l'égide de ce COS.

\section{Accréditation des laboratoires}

En France, l'accréditation COFRAC du laboratoire est un repère de savoir bienfaire dans le domaine considéré. Elle est obtenue à la suite d'un audit qualité complet, gestion et technique, qui porte à la fois sur le manuel d'assurance de la qualité du laboratoire, qui regroupe les documents où sont définies et décrites les opérations concernant la gestion des échantillons, des équipements, des demandes d'essai, des résultats, et sur la compétence technique à réaliser les essais soumis à accréditation. Il doit bien évidemment être à jour de toutes les révisions de procédures et conforme aux prescriptions du guide ISO/CEI 25 (International Standard Organisation, 1990).

En ce qui concerne le mesurage de la radioactivité à des niveaux trouvés dans l'environnement, deux programmes d'accréditation COFRAC sont concernés : le programme $\mathrm{n}^{\circ} 135$ « Analyse des radionucléides dans l'environnement » et dans une moindre mesure le programme ${ }^{\circ} 99-4$ « Analyse des contaminants chimiques chez les animaux, dans leurs produits et les denrées alimentaires destinées à l'homme ou aux animaux ». Dans le cadre du programme 135, trois niveaux de radioactivité sont répertoriés, allant du domaine des traces à celui caractéristique de situations incidentelles, voire accidentelles. Les laboratoires accrédités COFRAC se doivent d'utiliser les documents normatifs AFNOR et ISO précisant les conditions de réalisation des mesurages. 


\section{Conclusion}

La contamination potentielle de l'environnement par des matières radioactives entraîne régulièrement des débats de société. Dans ce contexte, il est également aisé d'imaginer l'importance de pouvoir identifier et quantifier précisément une source de contamination de l'environnement, de préciser les apports respectifs de différentes sources, et/ou d'en exclure certaines. Dans ces situations, la qualité et la fiabilité des stratégies d'échantillonnage, la réalisation des prélèvements ainsi que les résultats de mesurage ne doivent pas être mis en doute par l'une ou l'autre des parties afin que les discussions restent dans le champ de l'interprétation des résultats et non dans celui de leur contestation. Le mesurage doit donc s'appuyer sur une méthodologie exploratoire du milieu et une métrologie reconnues et référencées, si possible dans des normes qui sont le résultat d'un consensus. En apportant une réponse technique sans ambiguité, toute expertise d'impact basée sur des échantillons représentatifs des niveaux environnementaux dont le contenu radioactif sera mesuré par l'intermédiaire d'un laboratoire d'essai certifié ou accrédité, gagne alors en crédibilité.

Dans le cadre institutionnel, à la demande des collectivités locales ainsi que des Ministères de la santé, de l'environnement et de l'aménagement du territoire et de l'industrie, mais aussi parfois sous la pression du public et des médias et dans un contexte de contentieux, des mesurages contradictoires peuvent être réalisés par plusieurs laboratoires sur des échantillons de l'environnement. Dans certains cas, des divergences relativement importantes entre les résultats ont été constatées. Elles ont été expliquées par le manque de standardisation des méthodes et des moyens de prélèvement et de mesurage utilisés par les laboratoires.

Le développement de la normalisation dans le domaine de la mesure de la radioactivité, en particulier au plan français, est donc significatif d'une volonté de tous les acteurs de partager leur savoir-faire et d'améliorer la transparence dans leur domaine. Cet objectif de transparence est également conforme à la volonté des pouvoirs publics. La disponibilité de ces normes permet aujourd'hui de les intégrer comme outil de décision. Un des premiers objectifs de la commission M60-3 est donc d'achever un corpus de normes sur le mesurage des radionucléides présents au sein des différents compartiments de l'environnement air, eau, sol et indicateurs biologiques, ainsi que de traiter cet aspect pour les aliments.

Notons le caractère parfois innovant des travaux réalisés par la commission, notamment ceux sur les bioindicateurs. Ce corpus de normes constituera un ensemble sans contre partie au plan international. La décision a donc été prise, notamment par le BNEN et l'AFNOR, de proposer l'internationalisation de ces normes, c'est-à-dire leur reconnaissance au niveau de l'ISO. En 1999, le jeu 
de normes relatif aux mesurages des radionucléides dans les sols a été soumis au vote de l'ISO comme nouveau sujet d'étude à traiter au sein de l'ISO/TC 85/SC 2/WG 17. Les pays membres de l'ISO ayant retenu le projet, la première revue des projets français a été réalisée en mai 2000 lors de la réunion suivante de ce groupe de travail. Les projets sont aujourd'hui en circulation au niveau du sous-comité 2 . Pour continuer dans cette voie, la prochaine soumission à l'ISO devrait porter sur les normes traitant du mesurage des radionucléides dans l'eau, avec notamment la prise en compte de l'existant au plan ISO. Dans l'ensemble, il faut souligner le niveau soutenu de l'intérêt porté à ces travaux au plan international démontrant, s'il était besoin, l'importance et l'utilité des sujets traités pour des préoccupations qui dépassent le champ national.

\section{RÉFÉRENCES}

Académie des sciences (1996) Quelle place pour la métrologie en France à l'aube du XX $X^{\mathrm{e}}$ siècle ? Rapport commun Académie/CADAS n 5. Editions Tec \& Doc, Paris.

CCE (1998) Directive 98/83/CE du conseil du 3 novembre 1998 relative à la qualité des eaux destinées à la consommation humaine. Journal officiel $n^{\circ} \mathrm{L} 330 \mathrm{du} 05 / 12 / 1998$.

Ibrahim S.A., Webb S.B., Kattel A. (1995) Sources of misinterpretation for environmental plutonium measurements, J. Radioanal. Nucl. Chem. 194 (1), 213-219.

International Standard Organisation (1989) ISO 9698, Qualité de l'eau - Détermination de l'activité volumique du tritium - Méthode par comptage des scintillations en milieu liquide, $7 \mathrm{p}$.

International Standard Organisation (1990) ISO GUIDE 25, Prescriptions générales concernant la compétence des laboratoires d'étalonnage et d'essais, $7 \mathrm{p}$.

International Standard Organisation (1992) ISO 9696, Qualité de l'eau - Mesurage de l'activité alpha globale dans l'eau non saline - Méthode par source concentrée, $11 \mathrm{p}$.

International Standard Organisation (1996) NF EN ISO 14001, Systèmes de management environnemental, $15 \mathrm{p}$.

International Standard Organisation (1997) ISO 10703, Qualité de l'eau - Détermination de l'activité volumique des radionucléides par spectrométrie gamma à haute résolution, $17 \mathrm{p}$.

Laitinen (1973) The seven ages of an Analytical Method, Anal. Chem. 45 (14).

National Council on Radiation and Measurements (1994) A Handbook of Radioactivity Measurements Procedures, NCRP Report $n^{\circ} 58,3^{\mathrm{c}}$ rev.

National Radiological Protection Board (1995) Rapport technique, Vol. 6(1).

Nir-El Y. (1998) Application of reference materials in the accurate calibration of the detection efficiency of a low-level gamma-ray spectrometry assembly for environmental samples, $J$. Radioanal. Nucl. Chem. 227 (1-2), 67-74.

OECD (1992) The OECD principles of Good Laboratory Practice, Environmental Monograph $\mathrm{n}^{\circ} 45$.

Parkinson J.A., Horril A.D. (1984) An assessment of variation due to laboratory and field conditions in the measurement of radionuclides, Nucl. Instrum. Meth. Phys. Res. 223, 598-60I.

Prichard E., Mackay G.M., Points J. (1998) Trace analysis: a structure approach obtaining reliable results, Royal Society of Chemistry.

Sima O., Dovlete C. (1997) Matrix effects in the activity measurement of environmental samples implementation of specific corrections in a gamma-ray spectrometry analysis program, Appl. Rad. Isot. 48 (1), 59-69. 